\title{
What makes a conscious process conscious?
}

Max Velmans, Goldsmiths, University of London, New Cross, London SE14 6NW, UK Email:m.velmans@gold.ac.uk; web: http://www.gold.ac.uk/psychology/staff/velmans/

Behavioral and Brain Sciences, (2014) 37:1, pp.43-44. (an open-peer commentary on Newell, B.R. \& Shanks, D.R. (2014) Unconscious influences on decision making, BBS, 37:1, pp. 1-61.)

ABSTRACT Newell and Shanks' critical review considers only a very limited sense in which mental processes can be thought of as either conscious or unconscious and consequently gives a misleading analysis of the role of consciousness in human information processing. This commentary provides an expanded analysis of conscious processing that also reveals the various ways in which mental processes are unconscious.

According to Newell \& Shanks, it now seems to be generally accepted that whether a process is conscious should be operationally defined in terms of whether one has reportable knowledge of it. Consequently, in their critical review of unconscious processing, this is the criterion that they apply. However, as I have noted in the pages of this journal and elsewhere (c.f. Velmans, 1991 a, b, 1993, 1996, 2009) the psychological and philosophical literature often confounds three distinct senses in which a process might be said to be conscious. It might be conscious:

(a) in the sense that one is conscious of the process

(b) in the sense that the operation of the process is accompanied by consciousness (of its results) and

(c) in the sense that consciousness enters into or causally influences the process.

In these publications I have also considered how these different forms of 'conscious processing' apply to every stage of human information processing, although I only have space in this brief commentary to deal with a few examples. Ask yourself what's conscious about "conscious verbal thought". We do not have introspective access to how the preconscious cognitive processes that enable thinking produce individual, conscious thoughts in the form of "inner speech." However, the content of such thoughts and the sequence in which they appear does give some insight into the way the cognitive processes (of which they are manifestations) operate over time in problem solving, thinking, planning and so on. Consequently such cognitive processes are partly conscious in sense (a), but only in so far as their detailed operation is made explicit in conscious thoughts, thereby becoming accessible to introspection and consequent report. As noted above, being conscious in sense (a) is the criterion that Newell \& Shanks adopt.

However, many psychological processes are conscious in sense (b), but not in sense (a)-that is, we are not conscious of how the processes operate, but we are conscious of their results. This applies to perception in all sense modalities. When consciously reading this sentence for example you become aware of the printed text on the page, accompanied, perhaps, by inner speech (phonemic imagery) and a feeling of understanding (or not), but you have no introspective access to the processes which enable you to read. Nor does one have introspective access to the details of most other forms of cognitive functioning, for example to 
the detailed operations which enable "conscious" learning, remembering, engaging in conversations with others and so on.

Crucially, having an experience that gives some introspective access to a given process, or having the results of that process manifest in an experience, says nothing about whether that experience carries out or controls that process. That is, whether a process is "conscious" in sense (a) or (b) needs to distinguished from whether it is conscious in sense (c). Indeed, it is not easy to envisage how the experience that makes a process conscious in sense (a) or (b), could make it conscious in sense (c). Consciousness of a physical process does not make consciousness responsible for the operation of that process (watching paint dry does not actually make it dry on the wall). So, how could consciousness of a mental process carry out the functions of that process? Alternatively, if conscious experience results from a mental process it arrives too late to carry out the functions of that process. For example, try reading the following sentence and note what you experience:

\section{"If we don't increase the dustmen's wages, they will refuse to take the refuse."}

Note that on its first occurrence in your phonemic imagery or "inner speech", the word "refuse" was (silently) pronounced with the stress on the second syllable (refuse) while on its second occurrence the stress was on the first syllable (refuse). But how and when did this allocation of stress patterns take place? Clearly, the syntactic and semantic analysis required to determine the appropriate meanings of the word "refuse" must have taken place prior to the allocation of the stress patterns; and this, in turn, must have taken place prior to the phonemic images entering awareness.

Note too, that while reading, one is not conscious of any of the visual processing or pattern recognition that is required to identify individual words, or of any syntactic or semantic analysis being applied to the sentence. Nor is one aware of the processing responsible for the resulting inner speech (with the appropriate stress patterns on the word "refuse"). The same may be said of the paragraph you are now reading, or of the entire text of this paper. You are conscious of what is written, but not conscious of the complex input analyses involved. And if you are not conscious of how these processes operate, in what sense can they be said to be under "conscious control"?

The same may also be said about the detailed operation of nearly every other form of human information processing (see reviews cited above). According to Newell \& Shanks, "evidence for the existence of robust unconscious influences on decision making and related behaviors is weak, and many of the key research findings either demonstrate directly that behavior is under conscious control or can be plausibly explained without recourse to unconscious influences" ( $p$ 19). On the contrary, evidence for the influence of unconscious mental processes on human behaviour is ubiquitous. Indeed, if these complex processes were conscious in the sense that Newman \& Shanks intend, they should be directly available to first-person introspection, in which case there would be no need to discover their operation by means of laborious, third-person, cognitive psychological research!

\section{REFERENCES}


Newell, B.R., and Shanks, D.R. (2014) Unconscious influences on decision making: A critical review. Behavioral and Brain Sciences, 37(1), 1-61.

Velmans, M. (1991a) Is human information processing conscious? Behavioral and Brain Sciences 14(4): 651-669.

Velmans, M. (1991b) Consciousness from a first-person perspective. Behavioral and Brain Sciences 14(4): 702-726.

Velmans, M. (1993) Consciousness, causality and complementarity. Behavioral and Brain Sciences 16(2): 404-416.

Velmans, M. (1996) Consciousness and the "causal paradox." Behavioral and Brain Sciences 19(3): 537-542.

Velmans, M. (2009) Understanding Consciousness, Edition 2. London: Routledge/Psychology Press. 